patients were treated with concurrent chemoradiation followed by brachytherapy. The median follow-up period was 47 months. The three-year DFS and OS were $13.3 \%$ and $13.3 \%$ respectively.

Conclusion CCCC has a poor prognosis, stage for stage compared to other histologies. The FIGO stage, tumour size, lymphovascular space invasion and pelvic node status were factors that predicted the prognosis. Adjuvant radiotherapy or chemoradiotherapy have a limited role in the treatment of this rare cancer.

Disclosures The authors have no potential conflict of interest to disclose.

\section{EFFICACY OF A MULTI-INGREDIENT CORIOLUS VERSICOLOR-BASED VAGINAL GEL IN HPV+ WOMEN OLDER THAN 40 YEARS: SUB-ANALYSIS OF PALOMA CLINICAL TRIAL}

${ }^{1}$ Yann Gaslain, ${ }^{2}$ Luis Serrano, ${ }^{3}$ Andrés Carlos López, ${ }^{2}$ Silvia González, ${ }^{4}$ Santiago Palacios, ${ }^{5}$ Damian Dexeus, ${ }^{6}$ Pluvio Coronado, ${ }^{7}$ Jesús de la Fuente, ${ }^{8}$ José Antonio López, ${ }^{9}$ Cristina Vanrell. ${ }^{1}$ Procare Health; ${ }^{2}$ Centro Médico Gabinete Velázquez; ${ }^{3}$ Hospital Quironsalud; ${ }^{4}$ Instituto Palacios Salud Y Medicina de la Mujer; ${ }^{5}$ Women's Health Institute; ${ }^{6}$ Hospital Clínico San Carlos; ${ }^{7}$ Hospital Universitario Infanta Leonor; ${ }^{8}$ Hospital General Universitario de Alicante; ${ }^{9}$ Hospital de la Santa Creu I Sant Pau

\subsection{6/ijgc-2020-ESGO.19}

Introduction/Background HPV clearance and resolution of cervical HPV-dependent lesions become difficult in peri and postmenopausal women. The objective of this sub-analysis was to evaluate the effect of the Papilocare ${ }^{\circledR}$, a multi-ingredient Coriolus versicolor-based vaginal gel, on the normalization of cervical HPV-dependent atypia (ASCUS and LSIL) and associated colposcopic alterations in women older than 40 years.

Methodology Paloma clinical trial (ClinicalTrials. gov NCT04002154) was a multicenter, randomized, open-label, parallel-group, usual practice-controlled clinical trial. Unvaccinated HPV positive women aged between 30-65 with cytology of ASCUS or LSIL and concordant colposcopic image were randomized into 3 groups: A) Papilocare ${ }^{\circledR} 1$ cannula/ day for 1 month +1 cannula/alternate days for 5 months; B) Papilocare ${ }^{\circledR} 1$ cannula/day for 3 months +1 cannula/ alternate days for 3 months; C) Control group: no treatment (usual clinical practice). Primary endpoint:\% of patients with normal cytology and concordant colposcopy after 6 months of treatment in the total population, high-risk HPV $(16,18,31,33,35,39,45,51,52,56,58,59,68)$ and very high-risk HPV (patients infected by any combination of 16, 18 and 31) subpopulations. Pap smear evaluations were blind and centrally conducted by an independent researcher at the IECM laboratory (Lugo, Spain). Papilocare ${ }^{\circledR}$ arms (A+B) were combined as treatment group.

Results A total of 41 out of 84 evaluated patients included in Paloma trial were older than 40y [mean (SD) age: 47.71 (5.56)], of which 30 and 13 were high-risk HPV and 16-18$31 \mathrm{HPV}$ patients, respectively. At 6 months, normal cytology and concordant colposcopic image was observed in 92\%, 90\% and $75 \%$ of patients treated with Papilocare ${ }^{\circledR}$ vs $50 \%, 33 \%$ and $40 \%$ of patients in control group, in the total population, and high-risk and 16-18-31 subpopulations $(\mathrm{p}=0.0066$; $\mathrm{p}=0.0031 ; \mathrm{p}=0.2929$, Fisher test) respectively.
Conclusion Papilocare ${ }^{\circledR}$ showed a robust efficacy in normalizing cervical HPV lesions in women older than 40 years old, with a statistically significant difference vs control group in the total and high-risk populations.

Disclosures

Funding Procare Health Disclosure: J.Cortés, S.Palacios, D. Dexeus, L. Serrano: Advisory/Consulting Role and Speakers at Procare Health. Y. Gaslain: CEO of Procare Health.

All other authors have declared no conflicts of interest.

\section{REAL-LIFE EFFICACY OF A MULTI-INGREDIENT CORIOLUS VERSICOLOR-BASED VAGINAL GEL IN HIGH- RISK HPV PATIENTS: INTERIM ANALYSIS}

${ }^{1}$ Yann Gaslain, ${ }^{2}$ Javier Cortés, ${ }^{3}$ Javier de Santiago, ${ }^{4}$ María Pilar Sanjuán, ${ }^{5}$ Marta Agenjo, ${ }^{2}$ Gabriel Fiol, ${ }^{6}$ Carmen Garcia, ${ }^{7}$ Marta Gurrea, ${ }^{2}$ Perla Hernández. ${ }^{1}$ Procare Health; ${ }^{2}$ Private Practice; ${ }^{3}$ Centro Oncológico MD Anderson; ${ }^{4}$ Clínica Ginemed; ${ }^{5}$ Hospital Sanitas La Zarzuela; ${ }^{6} \mathrm{Hm}$ San Francisco; ${ }^{7} \mathrm{Hospital}$ La Fe

\subsection{6/ijgc-2020-ESG0.20}

Introduction/Background Real-life studies inform on the 'effectiveness' of a treatment what is intended to do in routine circumstances. The aim of this study is to evaluate the efficacy of Papilocare ${ }^{\circledR}$ - a multi-ingredient Coriolus versicolor-based vaginal gel- on repairing high-risk (HR) HPV-dependent lowdegree cervical lesions and HR-HPV clearance in real-life practice.

Methodology Observational, multicenter, prospective, onecohort study (PAPILOBS study ClinicalTrial.gov: NCT04199260). Currently recruiting 300 vaccinated or not HPV-positive women aged $>25 \mathrm{y}$ with Pap smear of ASCUS or LSIL and concordant colposcopy during routine clinical visits in Spain. Patients are treated with Papilocare ${ }^{\circledR} 1$ cannula/ day for 21 days the first month +1 cannula/alternate days for 5 months. After this 6-month period, patients with altered cytology and/or HPV persistency are treated for a 6-month extension treatment period with the same dosage.

Interim analysis of HR-HPV patients with normal Pap smear and concordant colposcopy image (primary endpoint) and patient with HR-HPV cleared (patients with total clearance or partial clearance together with negative Pap smear and normal colposcopy) at $6 / 12$ months is presented. The study was approved by the ethical committee of Public University Hospital of Puerta de Hierro (Madrid). Informed consent was signed by all patients.

Results At 6 months, data of 148 and 146 patients for Pap smear/colposcopy and HR-HPV presence, respectively, were available. $67.6 \%$ of patients $(100 / 148)$ had negative Pap smear and concordant colposcopy. HR-HPV clearance was observed in $58.9 \%$ of patients $(86 / 146)$. Data of 46 and 44 patients included in the 6-month extension treatment period for Pap smear/colposcopy and HR-HPV presence, respectively, were available. At 12 months, 78.3\% (36/46) of patients had negative Pap smear and concordant colposcopy and HR-HPV clearance was observed in $70.5 \%$ (31/44). Considering all study period, $77 \%(114 / 148)$ and $72.6 \%(106 / 146)$ of patients repaired HR-HPV-dependent cervical lesions and cleared HRHPV, respectively.

Conclusion In this interim analysis, repairing of HR-HPVdependent low-degree cervical lesions and clearing HR-HPV, in real life conditions, was achieved after 6-month treatment 
with Papilocare $^{\circledR}$ (or extending it up to 12 -months if needed) in 3 out of 4 patients. These findings need to be confirmed upon study completion.

Disclosures Funding: Procare Health Disclosure: J.Cortés: Advisory/Consulting Role and Speaker at Procare Health. Y. Gaslain: CEO of Procare Health. All other authors have declared no conflicts of interest.

\section{EFFICACY OF A MULTI-INGREDIENT CORIOLUS VERSICOLOR-BASED VAGINAL GEL IN HIGH-RISK HPV+ PATIENTS: RESULTS OF DIFFERENT STUDIES}

${ }^{1}$ Yann Gaslain, ${ }^{2} J a v i e r$ Cortés, ${ }^{3}$ Damian Dexeus, ${ }^{4}$ Santiago Palacios, ${ }^{5}$ Clara Gajino, ${ }^{2}$ Giovanni Miniello, ${ }^{6}$ Margarita Riera, ${ }^{7}$ Elena Marin. ${ }^{1}$ Procare Health; ${ }^{2}$ Private Practice; ${ }^{3}$ Women's Health Institute; ${ }^{4}$ Instituto Palacios Salud Y Medicina de la Mujer; ${ }^{5}$ Hospital Materno Infantil Teresa Herrera; ${ }^{6}$ Institut Catala de la Salut; ${ }^{7}$ Hospital Alvaro Cunqueiro

\subsection{6/ijgc-2020-ESGO.2}

Introduction/Background To evaluate the consistency of the efficacy of a non-hormonal multi-ingredient Coriolus versicolor-based vaginal gel, Papilocare ${ }^{\circledR}$, on HPV clearance in patients infected by high-risk HPV (HR-HPV) in several studies.

Methodology Results at 6 months from independent observational non-comparative studies carried out in three different public centers and in a one private center were compared to results from both a randomized, open, parallel and controlled clinical trial comparing the Papilocare ${ }^{\circledR}$ vs wait and see approach (The Paloma RCT) and a observational, multicenter, prospective, one-cohort study (Papilobs real-life study). Two prospective (Vigo and Bari studies) and two retrospective studies (Coruña and Hospitalet studies) have been performed.

Vigo study: HPV clearance of 25 patients infected by HPV 16 and/or 18 was evaluated as a secondary endpoint.

Bari study: HPV clearance of 98 HR-HPV patients was evaluated as primary endpoint.

Coruña study: 57 medical records of patients with HRHPV were analyzed. HPV clearance was evaluated as primary endpoint.

Hospitalet study: Data of 91 HR-HPV patients were evaluated. Primary endpoint: composite efficacy variable (percentage of patients with normal cytology and/or HPV clearance).

Papilobs study: Interim data of $148 \mathrm{HR}-\mathrm{HPV}$ patients is presented. HR-HPV clearance was evaluated as secondary endpoint.

Paloma RCT: 66 HR-HPV patients were evaluated. Percentage of patients with HR-HPV clearance was assessed as a secondary endpoint.

Results After the 6-month treatment period, 48\% and 57\% of patients cleared HPV 16-18 and HR-HPV in Vigo and Bari studies, respectively. A reduction of $58 \%$ was observed in number of HR-HPV patients (Coruña) and $72.5 \%$ of patients negativized cytology and/or cleared HR-HPV (Hospitalet) $(p \leq 0.0001$ vs baseline for all results, Chi-square). In the Paloma RCT, HR-HPV clearance was observed in $63 \%$ of patients treated with Papilocare ${ }^{\circledR}$ vs $40 \%$ in the control group. Similar rate of 59\% HR-HPV clearance was observed in the interim analysis of the Papilobs study.

Conclusion Papilocare ${ }^{\circledR}$ has shown significant and consistent rates of HR-HPV clearance ranging from $50 \%$ to $70 \%$ in the 6 different studies. This high consistently rate of HR-
HPV clearance should be further confirmed in ongoing studies.

Disclosures Funding: Procare Health. Disclosures: J.Cortés, S. Palacios, D. Dexeus, L. Serrano: Advisory/Consulting Role and Speakers at Procare Health. Y.Gaslain: CEO of Procare Health. All other authors have declared no conflicts of interest.

\section{RATIONALE AND EVIDENCE FOR EMERGING ANTIBODY- DRUG CONJUGATES IN GYNECOLOGICAL CANCERS: EFFECT OF ONLINE EDUCATION ON CLINICIAN KNOWLEDGE}

${ }^{1}$ Geoff Fisher, ${ }^{1}$ Amy Furedy, ${ }^{1}$ Juliette Vandenbroucque, ${ }^{2}$ Nicole Concin. 'Medscape Global Education; ${ }^{2}$ Medical University of Innsbruck

10.1136/ijgc-2020-ESGO.22

Introduction/Background A number of targeted antibody drug conjugates (ADCs) are emerging with the potential to become important new treatment strategies for gynecological cancers, including recurrent/resistant ovarian and cervical cancers. This study determined whether online continuing medical education could improve the knowledge of oncologists and obstetricians/ gynaecologists (obs/gyns) on the rationale and evidence for emerging ADCs.

Methodology A 30-minute online video lecture was launched for physicians outside the USA in December 2019. Data was collected to March 2020. Educational effect assessed with repeated-pairs pre-/post-activity, where individual participants served as their own control. 3 multiple-choice, knowledge questions and 1 self-efficacy, 5-point Likert scale confidence question were analyzed. Chi-squared test assessed pre- to postactivity change $(5 \%$ significance level, $\mathrm{P}<.05)$. Magnitude of change in total number of correct responses overall, and for each question, were determined with Cramer's V $(<.06=$ Modest, $0.06-0.15=$ Noticeable, $.16-.26=$ Considerable, $>.26=$ Extensive).

Results 49 oncologists and 154 obs/gyns completed pre- and post-activity questions. A positive educational effect was observed for oncologists (considerable effect, $\mathrm{V}=.217$, $\mathrm{P}=.0002$ ) and obs/gyns (noticeable effect, $\mathrm{V}=.097, \mathrm{p}=.0028$ ) with average $\%$ of correct responses increasing 40 to $62 \%$ for oncologists and 34 to $43 \%$, for obs/gyns. Participants with $3 / 3$ answers correct increased from pre- to post-activity (6 to $35 \%$ for oncologists and 6 to 12\%, for obs/gyns). Improvements in $\%$ of correct responses post-activity were seen for all 3 knowledge-based questions on antigen targets, and key trial data for tisotumab vedotin and mirvetuximab vedotin $(88 \%$, $39 \%$, 45\% improvements for oncologists; 70\%, 15\%, 16\% improvements for obs/gyns). Confidence in knowledge of ADCs also improved post-activity with a total average confidence shift of $38 \%$ for oncologists and $32 \%$ for obs/gyn. $62 \%$ of oncologists' and $44 \%$ of obs/gyns' responses were reinforced or improved post-activity. 34\% of all participants stated they would modify treatment plans as a result of participation in the activity.

Conclusion This on-demand, online video lecture resulted in a positive education effect for both oncologists and obs/gyns. However, persistent knowledge gaps are evident, especially amongst obs/gyns, suggesting there is a need for additional education as data on ADCs continues to emerge. Online medical education is valuable in establishing improved knowledge 